\title{
PERCEPCIÓN DE LOS USUARIOS SOBRE LA CALIDAD DEL SERVICIO DE ATENCIÓN EN VENTANILLA DEL DEPARTAMENTO DE REGISTRO DE LA UNIVERSIDAD NACIONAL
}

Yolanda Acuña Castro

Resumen Este artículo versa sobre la percepción que tiene el usuario sobre la calidad del servicio que se brinda en el ärea de atención al público en el Departamento de Registro de la Universidad Nacional. Es importante destacar que se concluye que el servicio que se brinda es bueno, no obstante se determina que existen serios problemas en cuanto al apoyo logístico se refiere. Asimismo se presenta una propuesta de capacitación para los funcionarios de dicho Departamento.
Luis R. Picazo

\section{Introducción}

La crisis de la calidad en los años 80 obligó a muchas empresas e instituciones a replantear el enfoque de la gestión de la calidad. Esto hace que las organizaciones se preocupen por la calidad del producto que se ofrece al cliente, debido a las exigencias cada vez mayores de éstos y a la necesidad de sobrevivencia en un ambiente cada vez más competitivo, donde se debe velar tanto por el bien como por el servicio que se brinda.

En este sentido, las universidades estatales y en particular la Universidad Nacional debe cuestionarse en qué medida el servicio que brinda el Departamento de Registro, responde a las necesidades actuales de los usuarios, considerando que esta dependencia es el primer contacto que los estudiantes y público en general tienen con la Universidad Nacional y que de ese servicio dependerá la imagen que se lleven de esta institución.

$\mathrm{El}$ contexto actual en que se encuentran inmersas las instituciones públicas, 
caracterizado por una serie de retos impuestos por la globalización, los recursos limitados y la vinculación con el sector productivo, entre otros, exigen a las organizaciones buscar formas más eficaces de hacer las cosas, dado el incremento de la competencia por parte de diferentes instituciones privadas.

Ante esta situación, las universidades estatales, se enfrentan a la competencia que surge como resultado del incremento de universidades privadas, las cuales se han hecho sentir de forma muy agresiva y con estrategias muy novedosas de gestión universitaria, en las que se manifiesta la preocupación por brindar un servicio de calidad a sus estudiantes para satisfacer sus necesidades.

Por tanto, para satisfacer y superar las expectativas de los usuarios en relación con el servicio que brinda el Departamento de Registro, la Universidad Nacional debe conocer y preocuparse por las necesidades o exigencias reales que tienen los usuarios preguntándose: ¿ En qué medida el servicio que se presta responde a esas necesidades?

En esta perspectiva, es que se definió como problema de investigación el siguiente: Determinar la calidad del servicio de atención al usuario en ventanilla, en el Depto. de Registro de la Universidad Nacional.

De conformidad con eI problema planteado se formularon los siguientes objetivos:

Evaluar el servicio de atención al usuario que brinda el Departamento de Registro de la Universidad Nacional, en ventanilla, basado en las actuales normas y principios de servicio al cliente, durante el período de febrero a julio de 1998.

Elaborar una propuesta de un Plan de Capacitación que sirva de base para el desarrollo de una capacitación sistemática y permanente para los funcionarios del Departamento de Registro de la Universidad Nacional.

Con el propósito de dar sustento a los objetivos de esta investigación, se consideró necesario analizar algunos aspectos conceptuales que se relacionan con el tema de la filosofía de calidad en el servicio al cliente. Asimismo se pretende dar algunos elementos sobre lo que ha sido la práctica institucional en este campo.

\section{Aspectos metodológicos}

1. Tipo

Esta investigación es de carácter descriptivo, dado que se recopiló y analizó la información, con el propósito de comprender, describir y presentar una propuesta de solución a los problemas detectados. Está relacionada con condiciones y concepciones existentes con prácticas que generan actitudes que se sienten y tendencias diversas sobre la calidad del servicio que se brinda en la ventanilla en el Depto de Registro de la Universidad Nacional.

\section{Población y muestra}

La población estuvo constituida por los usuarios del servicio de ventanilla que se presentan al Depto. de Registro y todos los funcionarios administrativos que ocupan puestos de jefatura en el Depto de Registro, así como la Directora del Departamento y la Vicerrectora Académica.

En relación con los usuarios, se consideraron en la muestra, los que se presentaron a la ventanilla a solicitar algún servicio en los meses comprendidos. Se consideró un 5\% de éstos, lo que significa que se les aplicó a un total de 50 usuarios. Es importante destacar que según (Goméz, 1992, p. 104) entre más homogénea sea una población menor es la muestra que se requiere para obtener conclusiones de un cierto grado de confianza.

Asimismo se consideraron en la muestra todos los funcionarios que ocupan puestos de atención en la ventanilla, así como los funcionarios que ocupan puestos de jefatura dentro de las secciones involucradas en servicios, a saber: admisión, sección empradronamiento y matrícula e historial académico. Asimismo a la Directora del Depto. de Registro y 
a la Vicerrectora Académica. En total la población esta constituida de 8 funcionarios que ocupan diferentes rangos en la Institución, escogidos por conveniencia como se indica:

A la Directora del Departamento de Registro, por ser la autoridad responsable de brindar el servicio en esa dependencia.

Tres funcionarios universitarios que prestan servicio al usuario en la ventanilla del Depto. de Registro.

Siete funcionarios que laboran como jefes en las diferentes secciones a saber: Sección de Servicio a la Administración docente, Sección de Matrícula y Sección de Historial Académico, al Subdirector y al Analista de Sistemas que ocupa un puesto de asesor de la Dirección por estar todos ellos directamente relacionados con la información que se genera en ventanilla.

El tipo de muestreo que se utilizó fue por conveniencia ya que se trató de usuarios que llegaron a la ventanilla del Depto. de Registro de la Universidad Nacional, en el período en que se realizaron las encuestas.

Se les aplicó una encuesta, en diferentes momentos del día, a saber: en la mañana y en la tarde, y en la noche los días miércoles, durante dos semanas en el mes de mayo y dos semanas en el mes de julio.

\section{Fuentes de información}

La recopilación de la información se efectuó por medio de la consulta a las siguientes fuentes: a) Informes y documentos de la Vicerrectoría Académica y del Departamento de Registro, b) Encuestas y Entrevistas aplicadas sobre esta problemática por instancias de la universidad, c) Bibliografía sobre calidad del servicio al usuario.

\section{Instrumentos}

En esta investigación se aplicaron una serie de instrumentos, a saber: encuestas, entrevistas no estructuradas y la técnica de observación directa.
Las encuestas fueron aplicadas a los usuarios del servicio a efecto de conocer la percepción de éstos sobre la calidad del servicio que se les brinda en el área de atención al público en el Departamento de Registro. También se aplicaron a los funcionarios que brindan servicio de atención al público y a los jefes de sección del Departamento de Registro.

Entrevista no estructurada con preguntas abiertas, aplicada a la Directora del Departamento de Registro, con el fin de conocer el criterio sobre la calidad del servicio que se brinda en el Departamento, así como conocer sobre las políticas y normativas sobre esta materia, que existen en la Universidad Nacional.

Técnica de observación directa en el área de atención al usuario, con el propósito de observar las instalaciones donde son atendidos los usuarios, las condiciones de espacio físico, en general el apoyo logístico con que cuenta el personal que brinda el servicio.

\section{Recolección y análisis de los datos}

La recolección de datos se desarrolló en las oficinas centrales del Departamento de Registro, en la sede central de Heredia, en el período comprendido del 4 de mayo al 31 de julio de 1998.

El instrumento fue administrado personalmente por la investigadora, en forma individual, a cada uno de los usuarios del servicio así como a los funcionarios de la Universidad, en donde se garantizó la confidencialidad de la información aportada. Como el instrumento fue aplicado directamente por el investigador, se recogí un $95 \%$ de los cuestionarios.

El análisis de la información se realizó con base en el paquete estadístico SPSS. La información se presenta en gráficos para facilitar su comprensión. Asimismo esta información según el procedimiento descrito, permitió establecer conclusiones y recomendaciones, que le servirán a la institución para tomar acciones y decisiones en el mejoramiento de la calidad del servicio que se brinda en el Departamento de Registro. 


\section{Marco conceptual}

\section{Caracteristicas de la calidad en el servicio}

Los usuarios buscan ser atendidos de la mejor manera en una institución de servicio, por ello debe prestárseles la mayor atención a sus demandas y conocer sus necesidades y expectativas, para brindarles el servicio de calidad que ellos desean.

Los requisitos de un servicio deben estar claramente definidos en términos de caracteristicas que sean observables y estar sujetos a la evaluación del usuario. Se enumeran algunas de las características que debe poseer un servicio de calidad (Sanabria, 1995, pág.2).

El servicio debe ser personalizado y profesional. El que brinda el servicio debe estar en la mejor disposición de atender al usuario.

Los servicios son inseparables, primero se venden, luego se producen y consumen al mismo tiempo. Cuando una persona brinda un servicio ésta forma parte del servicio mismo, por lo tanto el proveedor del servicio como el cliente influyen directamente en el resultado.

Otra característica es la variabilidad, la calidad de éstos depende del proveedor del servicio y cuándo, dónde y cómo lo brinde. Para administrar la variabilidad y ejercer control sobre la calidad, se deben tomar en cuenta ciertas medidas: (Sanabria, 1995, pág. 3):

- Selección y control de personal para brindar un mejor servicio.

- Aplicación de programas de incentivos.

- Implementación de un sistema de sugerencias y quejas.

- Visibilidad y responsabilidad ante el cliente procurando que el empleado eleve el nivel de responsabilidad sobre la calidad del servicio brindado.

\section{Requisitos para la calidad y mejoramiento del servicio}

La calidad y la satisfacción del usuario son temas importantes que han recibido una creciente atención en todo el mundo y sobre todo en la actualidad, con motivo de la globalización de los mercados. Por esta razón, en los últimos años las organizaciones se han preocupado por la calidad del producto que se ofrece al cliente. Esto hace que las instituciones públicas o privadas, debido a las exigencias cada vez mayores de sus clientes y a la necesidad de sobrevivencia en este ambiente competitivo, velen tanto por el producto como por el servicio que se brinda al cliente.

Para que una organización pueda cumplir con su cometido de brindar un servicio de calidad, debe tener un sistema integrado por políticas, procedimientos, procesos y responsabilidades claramente definidas. Según (Sanabria, 1990, pág. 6) para que este tipo de sistema sea efectivo debe reunir los siguientes requisitos:

- Políticas, planes y procedimientos que clarifiquen cómo deben realizarse las labores en la empresa.

- Moral e integridad profesional de los funcionarios que prestan el servicio.

Que el personal que brinda el servicio se sienta motivado.

Realizar periódicamente auditorías.

\section{Mejoramiento de los servicios}

Cabe señalar que las auditorías son muy importantes para la mejora continua de los servicios, ya que en muchas de las ocasiones no se presta un servicio eficiente, no porque haya malos trabajadores sino, porque los sistemas son inadecuados. En este sentido son las autoridades de la organización las que deben preocuparse por evaluar los sistemas, conocer las necesidades y expectativas de sus clientes, la satisfacción de éstos con respecto al servicio recibido. De esa manera la empresa pueda lograr mayor competitividad. 
Es importante tener presente que si las organizaciones pretenden estar orientadas al usuario tienen que modernizarse e innovar las formas de relacionarse con éste.

En este sentido señala que las empresas orientadas al cliente tienen 7 fuerzas motoras que contribuyen a crear ventajas competitivas:

$\begin{array}{ll}\text { - } & \text { velocidad } \\ \text { - } & \text { estilos de vida del cliente } \\ \text { - } & \text { valor agregado } \\ \text { - } & \text { servicio al cliente } \\ \text { - } & \text { innovación } \\ \text { - calidad del producto }\end{array}$

Como consecuencia de la orientación hacia el usuario, se requiere una renovación en el comportamiento del recurso humano. Uno de los mecanismos apropiados que les permiten a las organizaciones esa renovación, es por medio de la capacitación.

\section{Capacitación y desarrollo}

En este sentido es importante recalcar que el vertiginoso avance científico-tecnológico, muestra que la mera instrucción del hombre ya no satisface los reclamos de una sociedad dinámica y en transición. (Chruden y Sherman, 1988, pág. 186), consideran que el desarrollo de los empleados es vital para el éxito de las organizaciones modernas, debido a los rápidos cambios en la tecnología que requiere de conocimientos y habilidades necesarios para enfrentarse a los nuevos procesos.

Estos nuevos procesos suponen que toda organización debe alcanzar determinados objetivos y metas, combinando recursos financieros, materiales y humanos. De estos recursos el más valioso es el recurso humano porque es el que dinamiza los procesos.

Es así como la Ingeniería de Servicios plantea que las empresas generalmente necesitan tanta inversión o más en la gente pensante que en máquinas y equipo. La gente operativa de línea caracterizada por su de- sempeño mecánico y rutinario se transforma en gente pensante que soluciona problemas y toma decisiones en su relación de servicio al usuario. El reclutamiento y la seleción de personal es importante en todos los niveles.

La actuación del personal de línea en contacto con el cliente se mide no sólo por la productividad basada en reducir costos, sino más bien en su desempeño para crear clientes satisfechos que regresen a la empresa.

En síntesis, lo importante de la capacitación, es que debe existir compatibilidad entre las necesidades de la organización y las necesidades del funcionario, para que ambas partes obtengan el desarrollo esperado.

\section{Evaluación de la calidad del servicio}

El papel que desempeña el cliente tanto antes, durante y después de recibir el servicio no se debe obviar al realizar una evaluación del mismo.

Según (Sanabria, 1995, pág. 6) es necesario realizar una evaluación periódica en la que se contemple.

\section{- Estudiar el mercado}

- Verificar que el diseño de servicio se ajuste a lo que los clientes buscan y necesitan.

- Asegurarse que el servicio está acorde con lo diseñado.

- Evaluar el seguimiento a los reclamos, quejas y orientación del cliente en relación con el uso del servicio.

La evaluación periódica de la calidad del servicio sirve de retroalimentación y le permite a la organización tomar decisiones con el objeto de mejorar la calidad del servicio y de esa manera poder satisfacer las necesidades de los usuarios.

En este sentido, es importante que toda organización que realmente esté orientada al servicio al cliente, cuente con los recursos físicos idóneos que le permitan realmente ofrecer un servicio de calidad. Según (INTECO, 1993, pág. 5), los factores que 
deben considerarse en la evaluación de la calidad de los servicios son: Instalaciones físicas, cantidad de funcionarios que brindan servicio, cantidad de materiales, tiempo de espera, tiempo de prestación, tiempo de proceso, higiene, confort, tecnología.

\section{Marco institucional y quehacer del Departamento de Registro}

La estructura orgánica de la Universidad Nacional está conformada por: Asamblea Universitaria, Asamblea de Representantes, Consejo Universitario, Rectoría, Vicerrectoría Académica, Vicerrectoría de Vida Estudiantil y Vicerrectoría de Desarrollo, Facultades, Centros, Unidades Académicas y para-académicas e institutos.

La investigación se centró en la función de la Vicerrectoría Académica, instancia que dirige, reglamenta, coordina y evalúa la función del Departamento de Registro. Dentro de la estructura propia de la Vicerrectoría Académica, se contemplaron las tres direcciones de área, a saber: docencia, investigación y extensión. El Departamento de Registro se encuentra dentro de la estructura de la Dirección de Docencia.

El Departamento de Registro es una unidad para-académica adscrita a la Vicerrectoría que coopera con la atención integral del estudiante y participa en la administración de los sevicios docentes y estudiantiles. Su misión es "participar en la administración de la actividad docente, proporcionando un servicio al usuario con excelente calidad, óptimo y confiable, guiados por nuestros valores, el aspecto humano de la universidad y el mejoramiento continuo de los procesos, utilizando la mejor tecnología disponible" (Plan de Trabajo, Depto de Registro, 1998).

Su objetivo principal es "coadyuvar con las Unidades Académicas en la administración de los servicios docentes y registrar en custodia para dar fe del historial académico que se genera por la permanencia del estudiante en la Universidad, con eficiencia y eficacia." (Plan de trabajo, 1997).
Dentro de los objetivos fundamentales del departamento están:

Planear y ejecutar los procesos de admisión con base en el sistema y políticas de la institución.

Diseñar, planear y coordinar el proceso de matrícula.

Coordinar el trámite y registrar los resultados del reconocimiento y equiparación de estudios, grados y títulos.

Registrar, controlar y mantener actualizada la historia académica de los alumnos. Extender certificaciones y constancias de la historia académica del estudiante. Custodiar, organizar y mantener en el mejor estado los expedientes de los alumnos.

Extender el carné universitario a los alumnos regulares de la universidad.

Coordinar y colaborar en los procesos de graduación conjuntamente con las Facultades.

Elaborar la guía de horarios de acuerdo con la distribución de las aulas y la planta física disponible.

Registrar y mantener actualizado los planes de estudio ofrecidos por la Universidad.

Brindar información sobre los diferentes servicios que ofrece el Departamento en particular y la universidad en general.

\section{Servicios que brinda el Departamento de Registro}

Los servicios que ofrece este departamento son los siguientes:

Servicios a la administración docente: Brinda información para definir estrategias pedagógicas que pemitan dar seguimiento al estudiante desde que ingresa a la UNA hasta que se gradúa. Por otra parte la oferta docente debe estar claramente establecida y registrada de tal manera que alimente los diversos procesos de la administración docente. 


\section{Empadronamiento y matricula: Consta} de la inscripción y empadronamiento conforme a la demanda de la población estudiantil y a la oferta docente, la cual está definida en los planes de estudio de las diferentes carreras que ofrece la UNA de acuerdo con la estuctura curricular y los requisitos preestablecidos por cada unidad académica ajustados al calendario académico dentro del período lectivo. La determinación de grupos por cursos, asignación de profesores a los cursos, elaboración de horarios y ubicación de los grupos en las aulas.

Historial académico: Evalúa, registra y certifica el aprovechamiento del estudiante basándose en la información fidedigna que aporta el profesor. Toda la información académica debe estar contenida en el expediente del estudiante y mantenerla actualizada para facilitar los trámites de inscripción, expedición de certificaciones, conclusiones de estudios para efectos de graduación.

Atención al estudiante: ofrece atención integral al estudiante que le pemite evacuar las consultas y solicitudes en trámite para brindarle en un sólo lugar toda la información requerida.

Servicio informático: provee el soporte informático del Depto. de Registro, para lo cual debe contar con una tecnología avanzada acorde con las necesidades y naturaleza del servicio.

El Departamento de Registro tiene como objetivo fundamental brindar un servicio con excelente calidad, óptimo y confiable. Dentro de esta perspectiva, cabe señalar que de conformidad con información proporcionada por la Directora del Departamento, el área de atención al público, es el área más crítica que tiene el Departamento, dado que no existen políticas claras de la Vicerrectoría Académica, ni del propio Departamento, en lo que a la calidad del servicio se refiere.

Como bien lo señala (Berry, 1995, p. 48), un servicio de calidad proviene de un li- derazgo inspirado a lo largo de toda la organización, de una cultura corporativa orientada al cliente, de un excelente diseño del sistema de servicios, del uso eficiente de la información y la tecnología y de otros factores que se desarrollan lentamente en la organización. La calidad del servicio es el fundamento del mercadeo de servicios porque el producto central que se comercializa es un desempeño. El desempeño es el producto, es lo que el cliente adquiere.

Toda organización, debe preocuparse por realizar las actividades básicas que comprende la planificación de la calidad como son: Identificar los clientes y sus necesidades, desarrollar un producto que responda a esas necesidades, y desarrollar un proceso capaz de producir ese producto. (Jurán, 1990, pág 54).

En los últimos meses, el actual Vicerrector de Desarrollo, ha manifestado su interés de impulsar programas conducentes a levantar la imagen de la institución, y entre uno de los aspectos a mejorar es la calidad del servicio que se le brinda a los estudiantes en las diferentes instancias de la Universidad, específicamente en los departamentos de servicio, a saber Depto. de Registro, Depto. de Bienestar Estudiantil, Programa de Desarrollo Financiero, entre otros. Asimismo la Vicerrectora Académica, manifestó a principios del presente año, que se va a iniciar un trabajo orientado a mejorar la calidad del servicio en el Depto. de Registro, porque se han recibido muchas quejas por parte de la comunidad universitaria, con el servicio que actualmente se brinda.

De acuerdo con estas iniciativas, fue importante conocer el perfil del funcionario universitario que brinda el servicio de atención a los usuarios, en el Depto. de Registro a efecto de determinar en qué medida éste influye en el servicio que actualmente se brinda.

Asimismo se hizo necesario determinar si las condiciones de espacio físico como de apoyo logístico inciden igualmente en la calidad del servicio que se brinda, y que ha generado en las quejas de los usuarios. Todo ello, según lo manifiesta la propia Vicerrectoría de 
Desarrollo, tiene como objetivo satisfacer las necesidades y expectativas de los usarios, a efecto de que el servicio que se les brinde sea de su satisfacción.

\section{Resultados}

Los resultados que se obtuvieron de las encuestas que se aplicaron a los usuarios y funcionarios del Departamento de Registro, así como el resultado de algunas entrevistas que se realizaron a autoridades de la Universidad Nacional, dan cuenta de la situación real del servicio que brinda al público el Departamento de Registro.

El análisis de los datos se hizo dando respuesta a los objetivos planteados en la investigación, siguiendo el orden en que fueron presentados.

\section{Perfil del funcionario universitario que brinda el servicio de atención en ventanilla en el Departamento de Registro}

Al prestar un servicio es importante conocer el perfil de las personas que brindan el servicio, a efecto de determinar los conocimientos, actitudes y aptitudes de los mismos, para brindar un servicio de calidad.

Es por estas razones antes expuestas, que se considera importante analizar las generalidades de las personas que interactúan con el público. Los aspectos que se consideran son: nivel académico, tiempo de laborar en la Universidad Nacional, tiempo de laborar en el Depto. de Registro, capacitación recibida entre otros.

Estudios realizados: Los resultados que se obtienen mediante los instrumentos aplicados a los tres funcionarios que atienden directamente a los usuarios en las ventanillas del Depto. de Registro, revelan que un $100 \%$ han concluido estudios universitarios. Esto es importante no sólo porque se considera que se encuentran técnicamente capacitados para el desempeño de sus labores, sino porque se esperaria que su preparación académica les permita tener una visión más amplia de la importancia de sus funciones, de las necesidades y expectivas de los usuarios del servicio.

Es importante señalar que cada uno de ellos posee formación en: administración, secretariado profesional y educación respectivamente, carreras que están muy relacionadas con el puesto que desempeñan dichos funcionarios.

Gráfico 1

Estudios realizados por los funcionarios del Depto. de Registro
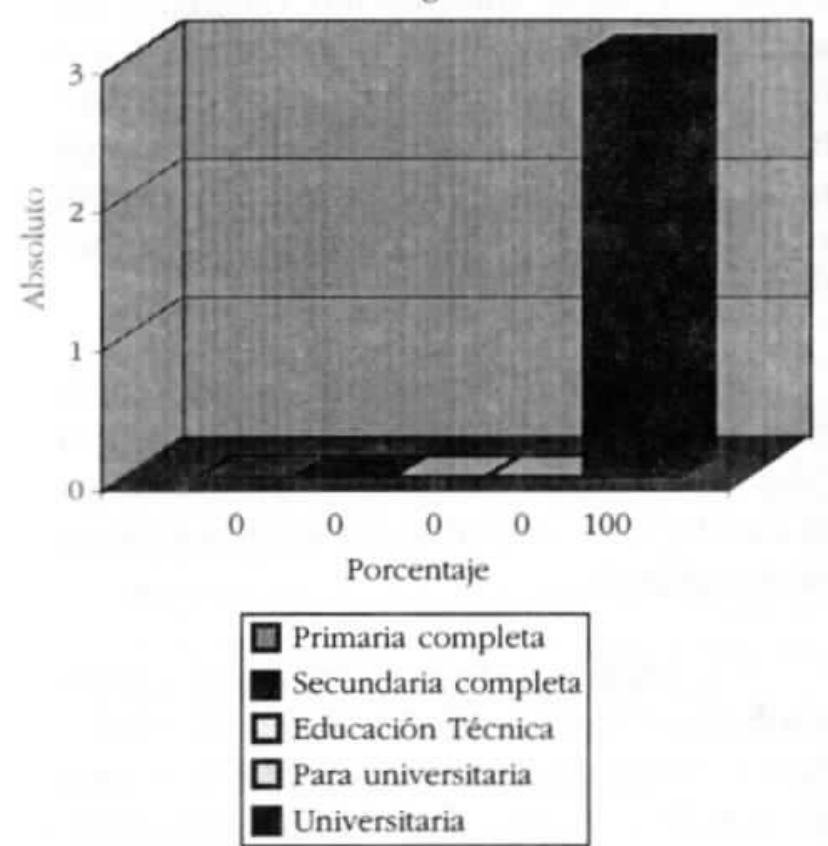

Gráfico 2

Tiempo de laborar en la Universidad Nacional

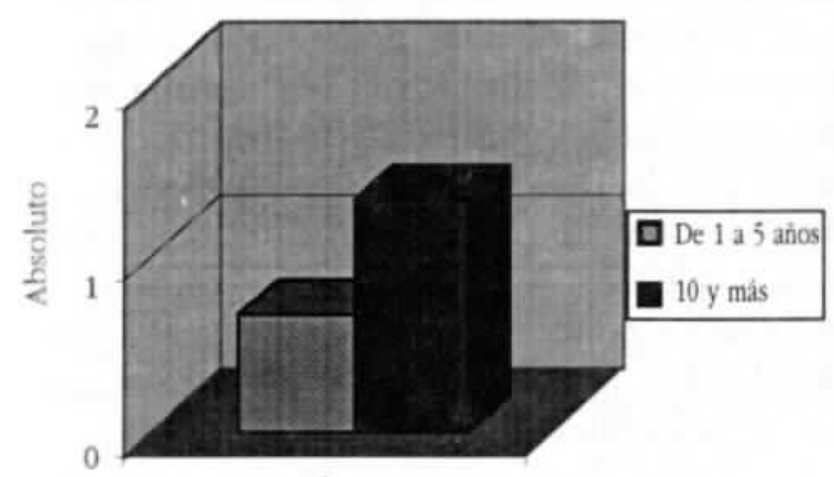

$33,3^{\circ} \quad 66,7$ Porcentaje 
Asimismo se puede observar en el gráfico número 2 , como un $66,7 \%$ de los funcionarios tienen más de 10 años de laborar para la Universidad Nacional, lo que demuestra que es un período de tiempo suficiente como para poder tener un conocimiento amplio de la institución. Es importante destacar que este es un requisito indispensable para estos puestos de ventanilla, dado que se requiere un conocimiento amplio de la institución por la diversidad de información que se solicita por parte de los usuarios. No obstante, cabe señalar que al indagar sobre el tiempo que tienen de laborar el puesto de atención al público, dos de ellos manifiestan tener de 1 a 5 años, lo que significa el $66,7 \%$.

\section{Gráfico 3}

Tiempo de laborar en el Depto. de Registro

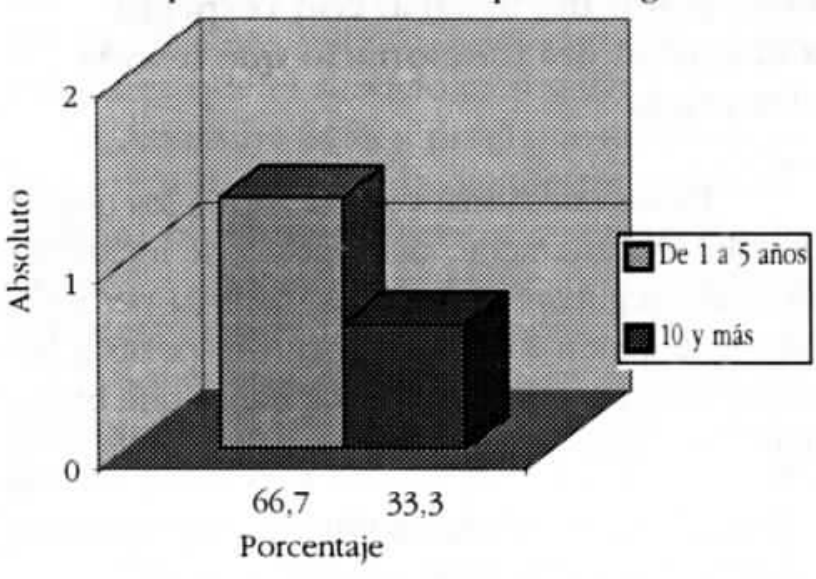

En relación con la clasificación de los puestos que ocupan, se denota que la clase de puestos es muy baja, dos de ellos ocupan el puesto de oficinista 3 y 1 de técnico en Administración 1.

Al preguntárseles sobre si consideran que sus puestos no se encuentran debidamente clasificados dos de ellos contestan que sí, significando un $66,7 \%$.

Capacitación recibida: Respecto a la capacitación recibida el $100 \%$ responde no haber recibido capacitación para el puesto que desempeña.

Como bien se puede ver en el desarrollo del presente trabajo, se hace énfasis en la capacitación como un elemento que ayuda a brindar un servicio de calidad y es un indicador que le permita al funcionario conocer y tener una visión más clara acerca de los objetivos y funciones dentro de la institución, de los procesos de trabajo y por supuesto como un instrumento para poder desempeñar más eficientemente sus labores.

Sobre este mismo tema, se preguntó a las jefaturas del Departamento si han fomentado la capacitación de los funcionarios, en el área del servicio al usuario, a lo que un $80 \%$ contesta afirmativamente. Sin embargo es importante señalar que aunque existe la mayor disponibilidad para que los funcionarios se capaciten, es mínima la capacitación que la institución brinda.

Como se desprende del análisis anterior, se puede decir que no se les ha brindado una adecuada capacitación a los funcionarios que dan servicio al público, a pesar de que la Institución cuenta con una Unidad de Capacitación y Desarrollo adscrita al Departamento de Recursos Humanos.

Se considera por tanto necesario que los funcionarios que atienden la ventanilla en el Departamento de Registro, puedan recibir capacitación en aquellas temáticas que tengan que ver con el servicio al cliente (Relaciones Humanas, Servicio al cliente, motivación y actitud personal, entre otros).

\section{Necesidades y expectativas del usuario del servicio de atención en la ventanilla del Departamento de Registro}

Con el objeto de conocer cuáles son las necesidades y expectativas del usuario, se indaga al respecto y las respuestas que se obtienen son las que se presentan a continuación:

Motivo de la visita: Para saber cuál de los servicios que brinda el Departamento de Registro es el que más utilizan los usuarios, se preguntó sobre el motivo de la visita ese día, los resultados que se obtienen se presentan en el gráfico No. 4. 
Gráfico 4

Motivo de la visita al Depto. de Registro

de la Universidad Nacional

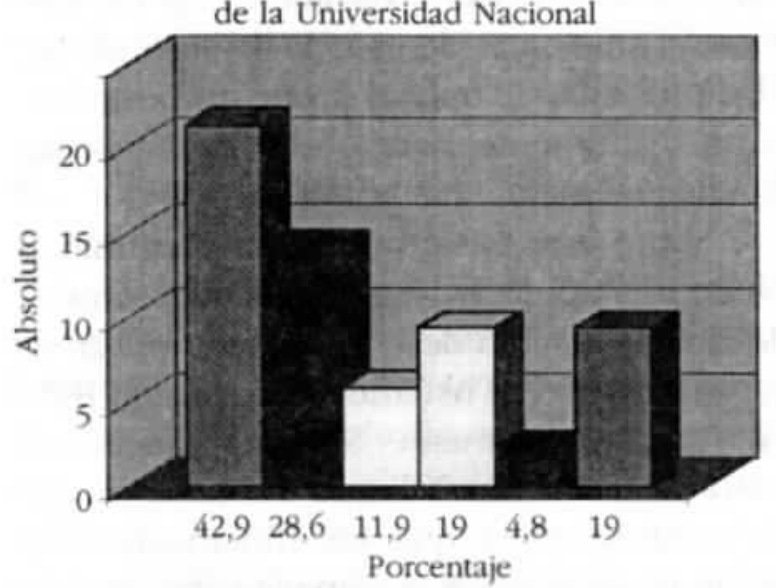

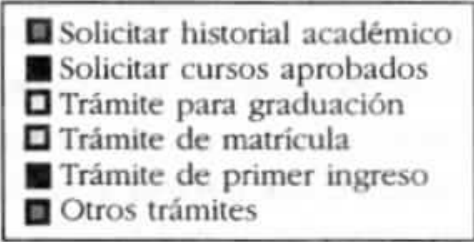

Los datos anteriores demuestran que un gran número de usuarios que visitan el Departamento vienen a solicitar el historial académico $(42,9 \%)$ y certificaciones de cursos aprobados $(28,6 \%)$ significando un $71,5 \%$ que vienen por el mismo servicio.

Criterio sobre la atención que se le brinda al usuario: Para conocer la valoración que el usuario le da al servicio de atención que se le ofrece en la ventanilla del Departamento de Registro se pregunta sobre la rapidez y confiabilidad del servicio recibido, las respuestas que se obtienen son las que se presentan a continuación:

\section{Gráfico 5}

Percepción del servicio que se brinda en el Área de Atención al púbico en el Depto. Registro

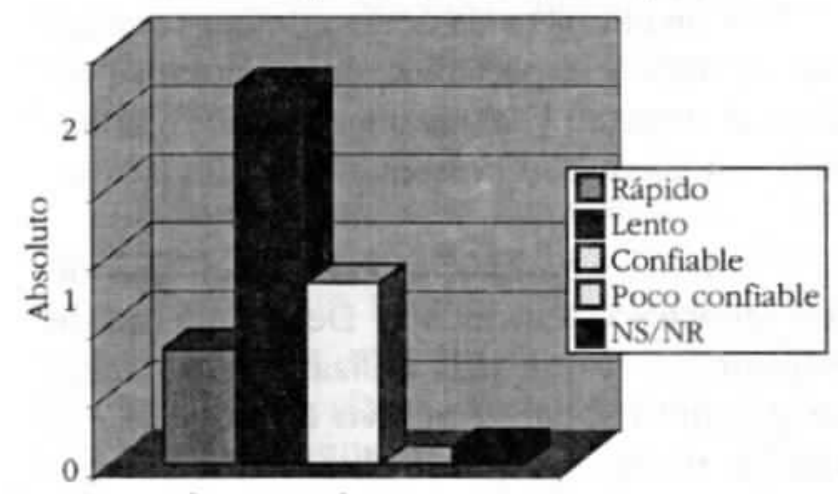

$\begin{array}{lllll}16,7 & 54,8 & 26,2 & 0 & 24\end{array}$ Porcentaje
Como se desprende del gráfico anterior el $54,8 \%$ considera que el servicio que se le brinda es lento, lo que provoca indisposición e insatisfacción en los usuarios que llegan al Departamento, lo que obviamente va en detrimento de la calidad del servicio que se brinda.

Pese a lo expuesto anteriormente un $26,2 \%$ de los usuarios indican que el servicio que se les brinda es confiable, lo que viene a compensar la lentitud del servicio que reciben.

En este sentido, es importante señalar que es necesario buscar las formas de hacer más rápido el servicio, sea abriendo mas ventanillas al servicio del usuario o analizando que es lo que hace el servicio lento y trabajar sobre este aspecto para mejorarlo.

\section{Percepción del usuario con respecto a la actitud del funcionario que brinda el servicio}

En los cuestionarios aplicados a los usuarios del Departamento de Registro, se incluyen algunas preguntas cuya fin es medir la percepción que tienen los usuarios con respecto a la actitud que presentan los funcionarios que brindan el servicio de atención en la ventanilla.

Gráfico 6

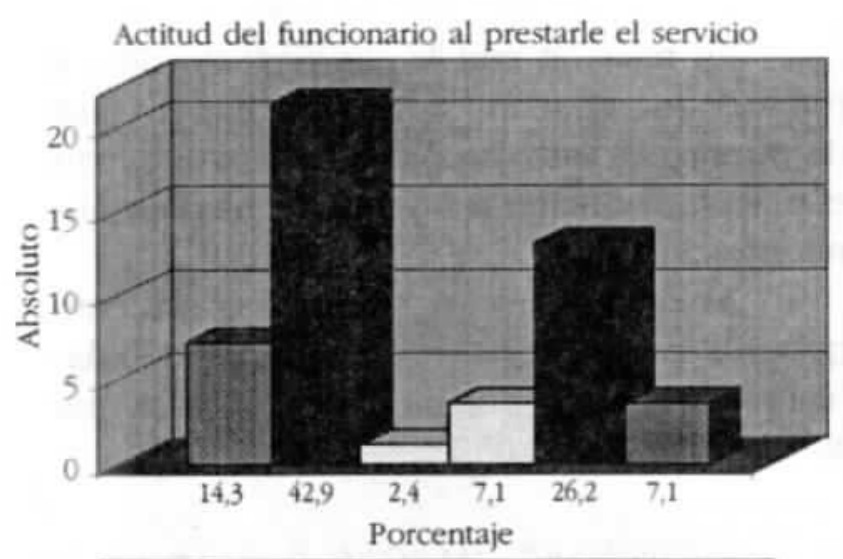

Al llegar lo saluda

Se preocupa por atenderlo de la mejor manera

Le ha ofrecido disculpas por la demora en la atención al servicio

Se despide de usted luego de ser atendido

Nada de las anteriores

NS/NR 


\section{Gráfico 7}

Percepción del servicio que se le brinda al usuario en el Depto. de Registro de la UNA

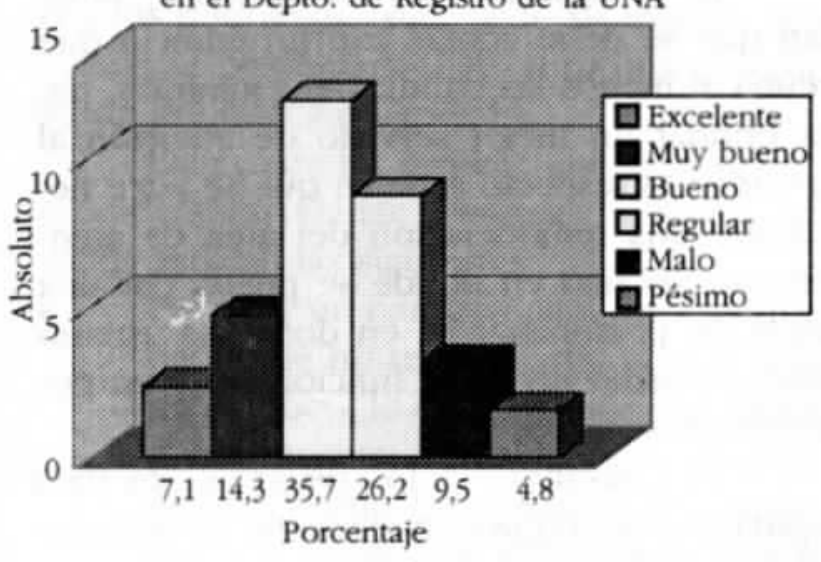

De acuerdo con los resultados que se obtienen se desprende que existe una percepción favorable del servicio que se brinda (50\%). Un 35,7\% considera que es bueno y un $14,3 \%$ muy bueno. Asimismo un $26,2 \%$ considera que el servicio es regular.

Asimismo se les pregunta a los usuarios sobre la actitud que demuestra el funcionario al prestarle el servicio y tenemos que un $42,5 \%$ contesta que existe preocupación por atenderlo de la mejor manera.

Sobre este mismo tema, se indaga a los funcionarios sobre cuál es su actitud al atender al público y un $100 \%$ contesta que se preocupa por atenderlos de la mejor manera.

Como se puede observar, existe coherencia entre lo que manifiestan los funcionarios y la perçepción de los usuarios.

\section{Condiciones de los recursos fisicos en lo que respecta al apoyo logístico del Departamento de Registro}

Con la finalidad de conocer cual es el apoyo logístico con que cuenta el Departamento de Registro, se les pregunta a los funcionarios al respecto, a efecto de determinar si existen algunas barreras que impiden la adecuada prestación del servicio. Las respuestas que se obtuvieron son las que se presentan en la siguiente información:
Barreras que impiden al funcionario del Departamento de Registro brindar un buen servicio

\section{Gráfico 8}

Barreras que impiden al funcionario del Departamento

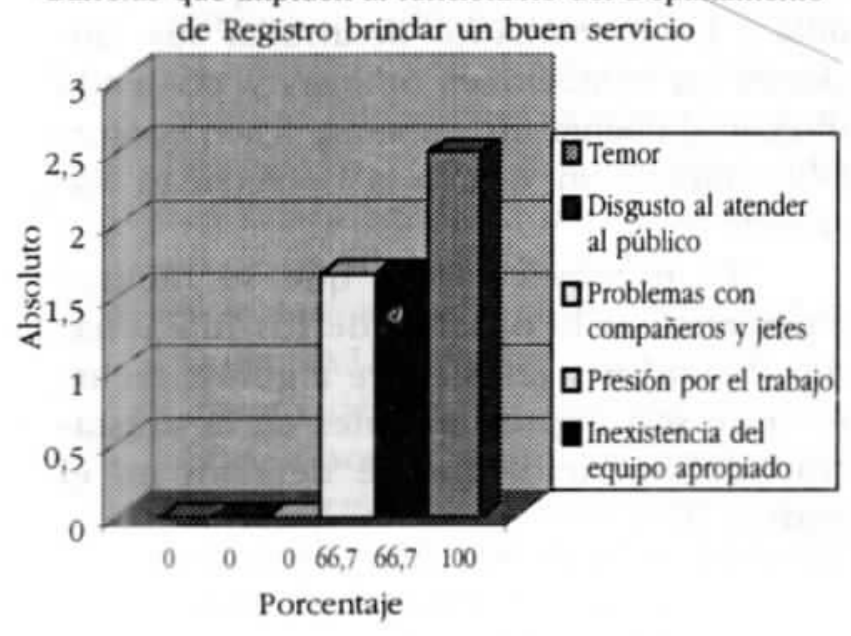

De la información anterior, se desprende que la principal barrera para brindar un buen servicio de atención, según los funcionarios consultados, es la falta de una infraestructura apropiada $(100 \%)$. Esto hace que al no contar con un espacio adecuado para la atención personalizada, provoca incomodidad y angustia, tanto a los funcionarios como a los usuarios. Además señalan que no existe suficiente equipo de cómputo para realizar sus funciones (sólo existen dos impresoras para tres ventanillas) lo que consideran hace más lento el servicio.

Se puede observar que las barreras que los funcionarios mencionan son subsanables y que esta responsabilidad es competencia de la Dirección del Departamento. Las barreras de equipo e infraestructura son las que más se mencionan. También los funcionarios mencionan que la presión del trabajo que ellos tienen son otro de los inconvenientes que deben afrontar al brindar el servicio y que por supuesto afecta la prestación del servicio.

Otros recursos que ayudarian a brindar un mejor servicio: Como se menciona 
anteriormente, para brindar un servicio de calidad que satisfaga las necesidades de los usuarios, es imprescindible no sólo contar con los recursos humanos capacitados y motivados, sino también con recursos materiales e infraestructura adecuada que le permita a los funcionarios desarrollar sus funciones en condiciones óptimas y por ende ofrecer al cliente un ambiente físico confortable durante su visita a la Universidad $\mathrm{Na}$ cional.

Es por esta razón, que se incluye en el análisis la opinión de los funcionarios, usuarios y jefes sobre algunos recursos que son imprescindibles en la prestación del servicio, que se detallan en el gráfico 9 .

\section{Gráfico 9}

Otros recursos que ayudarán a brindar un mejor servicio

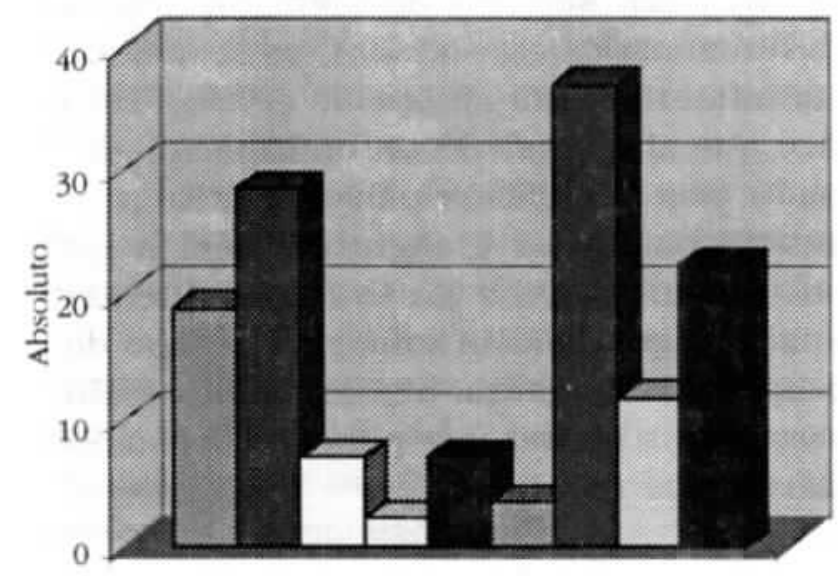

$\begin{array}{lllllllll}38,1 & 57,1 & 14,3 & 4,8 & 14,3 & 7,1 & 73,8 & 23,8 & 45,2\end{array}$ Porcentaje

\begin{tabular}{|l}
\hline Remodelación de las oficinas de servicio al usuario \\
Mejor distribución de las áreas para atención \\
personalizada \\
a Adecuada ventilación \\
Mejor iluminación \\
Salidas de emergencia \\
Parqueo para los usuarios \\
Adecuada rotulación de cada área de servicio \\
Asientos confortables para la espera \\
Servicios sanitarios accesibles
\end{tabular}

\section{- Estructura fisica:}

Al indagar con los funcionarios, señalan que se debe contar con un edificio que reúna al menos las condiciones mínimas, para brindar un mejor servicio de atención al usuario. Al respecto señalan que se hace necesario una remodelación del área de atención al público en donde se pueda dar una atención personalizada, en donde el usuario esté al mismo nivel del funcionario y en posición de sentado.

Al consultarle a los usuarios, un 95\% opina que se requiere mejoras en la estructura física $(38,1$ remodelación de las oficinas y $57.1 \%$ en mejor distribución de las áreas para atención personalizada).

Durante las visitas que realicé a esta área, se pudo constatar que efectivamente se hace necesario mejorar, dado que las ventanillas son muy altas, quedando el usuario muy distante del funcionario, cuya comunicación es a través de un agujero en el vidrio de la ventanilla. Asimismo el mostrador es muy incómodo y no permite la entrega y recibo de documentos ni tampoco poner las pertenencias que traen los usuarios (bolso, paquetes o sombrilla). Tanto los usuarios como los funcionarios del Departamento de Registro, coinciden que se debe hacer una mejora sustancial a la estructura física.

\section{Oficina de Información}

Al momento de realizada la investigación se determinó que no existe una ventanilla exclusiva de información lo que hace que los usuarios tengan que tomar ficha y hacer la fila para hacer alguna consulta.

Al consultar a las jefaturas del Departamento sobre esta situación, manifiestan que ellos se rotan cada semana para cubrir esa necesidad y se dispuso la ubicación de un escritorio en la sala de espera, para atender estas consultas.

Cabe agregar que a partir de enero de 1999, se dispuso ubicar una terminal de 
cómputo para uso de los usuarios, la cual permitirá hacer consultas a los estudiantes sobre los diferentes asuntos de su interés. Asimismo se prevé para 1999, abrir una ventanilla de información general.

Dentro de las mejoras que los usuarios consideran ayudarían a brindar un mejor servicios señalan las siguientes:

- Habilitar un parqueo para los usuarios, dado que no se cuentan con una zona de parqueo, lo que produce problemas y en ocasiones infracciones de tránsito, debido a que los usuarios se estacionan a la orilla de la vía pública, lo que provoca en ellos gran tensión, por el temor a sufrir un robo del vehículo o que sea golpeado, ya que el Depto. se ubica sobre la vía principal de acceso a la provincia herediana.

Asimismo un $14,3 \%$ de los usuarios encuestados opinan que se requiere mejor iluminación y salidas de emergencia, dado que el edificio solo tiene una vía de acceso, lo que en caso de alguna situación peligrosa pone en peligro la seguridad de los usuarios.

Un $73,8 \%$ de los consultados, opinan que se hace necesario una adecuada rotulación de cada área de servicio, porque no hay una señalización que facilite la ubicación de los usuarios en las diferentes ventanillas, lo que provoca muchas veces largas filas innecesarias. Un $45,2 \%$ de los usuarios, creen necesario la ubicación de servicios sanitarios accesibles dentro del edificio.

\section{v. Conclusiones}

Luego de analizar los resultados que se obtienen mediante la aplicación de los instrumentos, así como las entrevistas realizadas y la observación directa en el área de servicio al público del Departamento de Registro, en el periodo comprendido de marzo a julio de 1998, se establecen las siguientes conclusiones:
1. En relación con el perfil del funcionario que atiende las ventanillas del Departamento de Registro, la totalidad han concluido los estudios universitarios, en áreas afines al puesto que desempeñan: administración, secretariado profesional y educación. Este aspecto es importante, ya que permite al funcionario contar con la formación académica necesaria para el desempeño de las labores que realiza.

2. En lo que respecta al tiempo de laborar para la Universidad y para el Departamento de Registro en particular, la mayoría son funcionarios que tienen mucho tiempo de laborar para la institución al igual que para el Departamento de Registro.

Como se puede ver, es un personal que conoce a la institución y al Depto de Registro, por lo que puede estar en condiciones de brindar una información al usuario bastante precisa y completa.

3. En lo referente a la capacitación de los funcionarios encuestados, la Universidad Nacional y por ende el Departamento de Registro no cuenta con un programa de capacitación, sistemático y permanente, que responda a las necesidades de los funcionarios de este Departamento.

Específicamente las jefaturas y los funcionarios señalan que no existe una capacitación periódica de cursos sobre servicio al cliente, relaciones humanas, relaciones públicas, entre otros.

Cabe señalar que esta situación se presenta, a pesar de que existe una Unidad de Capacitación y Desarrollo que se encuentra adscrita al Depto de Recursos Humanos.

4. Con respecto a la capacitación sobre servicio al cliente se constata que únicamente han recibido algunas charlas a iniciativa de la propia Dirección del Departamento, pero que es insuficiente y esporádica. 
5. En relación con la percepción del usuario respecto al servicio de atención que se brinda en el área de atención al público del Depto. de Registro, un alto porcentaje considera que el servicio que se brinda es LENTO. A pesar de que el servicio es calificado como LENTO, los usuarios lo consideran CONFIABLE.

6. Es importante senalar que a pesar de que el servicio es lento, existe la percepción por parte de los usuarios, de que los funcionarios que brindan el servicio al público, se preocupan de atenderlos de la mejor manera. Por su parte los funcionarios que atienden ventanilla manifiestan que existe preocupación de su parte por atender a los usuarios de la mejor manera.

7. En relación con la percepción que tiene el usuario sobre el servicio que se les brinda, existe una mayoría que considera el servicio entre bueno y muy bueno.

8. En relación con el apoyo logístico con que cuenta el área de atención al público del Departamento de Registro, el mismo no reúne las condiciones necesarias para brindar un servicio de atención al público por cuanto:

\subsection{La falta de una infraestructura adecuada es la principal barrera para brindar un buen servicio de atención al usuario: opinión tan- to de los usuarios como de los funcionarios del Departamento.}

8.2. El espacio destinado para la atención de los usuarios, no tiene una apariencia agradable, lo que no ayuda a que las personas se sientan a gusto mientras esperan ser atendidos.

8.3. No existe una ventanilla exclusiva de información lo que ocasiona que los usuarios tengan que tomar ficha y hacer la fila para realizar alguna consulta breve.

8.4. El edificio no cuenta con servicios sanitarios accesibles a los usuarios para satisfacer las nece- sidades fisiológicas, lo que denota una vez más que las autoridades de la institución, no les interesa la satisfacción del usuario.

8.5. El área de atención al usuario no cuenta con sillas confortables y suficientes.

8.6. No existe una adecuada rotulación en las diferentes ventanillas.

8.7. No existe suficiente iluminación y ventilación.

8.8. No ofrece servicio de parqueo para los usuarios y funcionarios del Departamento de Registro.

9. En cuanto al equipo de cómputo con que cuenta el área de atención al públi$\mathrm{co}$, los funcionarios opinan (y se logra comprobar mediante la observación) que el mismo es escaso y desactualizado, lo que causa un gran problema de lentitud en la atención de los usuarios. Cabe señalar que las impresoras son compartidas por varios funcionarios, por lo que el servicio no puede brindarse con la eficiencia y eficacia que se quisiera.

10. Es importante señalar que una gran mayoría opina que sería conveniente que el servicio de cajas del Departamento de Gestión Presupuestario y Financiero se encuentre ubicado dentro del área de servicio al público del Depto de Registro, a efecto de ahorrarse tiempo en los trámites que realizan.

Con el objeto de complementar las conclusiones anteriormente señaladas, a continuación se indican algunas recomendaciones que se considera pueden contribuir a mejorar y fortalecer el servicio de atención al usuario que brinda el Departamento de Registro de la Universidad Nacional.

\section{Recomendaciones}

1. Es importante al momento de reclutar y seleccionar personal para puestos de atención directa con el público, 
considerar como un elemento fundamental para la selección, que a estas personas les satisfaga la relación personalizada con los usuarios.

2. Realizar un diagnóstico de necesidades de capacitación del personal, que permita identificar los requerimientos de capacitación que tienen tanto los funcionarios que atienden la ventanilla, como los demás funcionarios del Departamento.

3. Es fundamental, que las jefaturas de sección del Departamento, así como la Dirección del Departamento, participen en cursos de capacitación sobre temas como: Planeación Estratégica, los Recursos Humanos en la Empresa, La Calidad en el servicio al cliente entre otros, de tal manera que les permita una mayor identificación y compromiso con la calidad del servicio que brinda el Departamento, ya que son ellos los responsables de dictar las políticas y lineamientos que se deben seguir en la prestación del servicio.

4. A nivel institucional, del Consejo Universitario establezca políticas claras sobre la calidad del servicio al usuario, dado que en la institución no existen.

5. Uno de los principios de calidad es que los procedimientos estén claros y por escrito, a fin de facilitar la labor a los funcionarios que brindan el servicio. Por lo anterior, las jefaturas en coordinación con la Dirección y con la participación de los funcionarios, la revisión de éstos a efecto de actualizarlos y hacerlos del conocimiento de todos los funcionarios.

6. Con la finalidad de hacer más eficiente y rápido el servicio al usuario, se sugiere dotar al personal del área de atención al público de equipo de cómputo (computadoras, impresoras, programas) y equipo actualizado y rápido, que permita al funcionario brindar un servicio de atención eficiente.
7. Establecer un sistema que permita al Departamento de registro conocer y estudiar las quejas, sugerencias y recomendaciones tanto de los usuarios como de los funcionarios, de manera tal que este sistema sea una fuente importante de ideas, que ayude a mejorar cada el día el servicio que se brinda.

8. Se sugiere que el Departamento de Registro mejore aún más, la formulación del plan estratégico, donde se contemple una estrategia de atención al usuario, la misión y visión del Departamento, los objetivos y metas, la imagen que desean proyectar, las necesidades de capacitación, de infraestructura entre otros.

9. Se recomienda como elemento prioritario, una mejora en la estructura física del Departamento. Se sugieren las siguientes modificaciones:

- Remodelar totalmente el área de atención al público. (Incluye piso cerámico, diseño de muebles para atención personalizada y pintura).

- Mejorar sustancialmente la estructura física e incluir elementos como:

- Colocación de sillas confortables.

- Instalación de un T.V. y un VHS en la sala de espera.

- Reubicación de la pantalla númerica.

- Rotulación adecuada de todos los servicios.

- Reubicación pantalla electrónica.

- Instalación de un servicio sanitario para uso exclusivo de los usuarios.

- Instalar un buzón para sugerencias de los usuarios en un lugar visible.

- Construir al menos una salida de emergencia ya que solo se cuenta con una puerta para la entrada y salida de los usuarios, 
lo que ocasiona inseguridad y temor en los usuarios.

Asignar una área de parqueo tanto para los usuarios como para los funcionarios del Departamento, dado que por ubicación del edificio se genera mucho disgusto y temor de parte de los usuarios por la inseguridad en que quedan sus vehículos.

10. Ubicación de una ventanilla exclusiva de INFORMACION GENERAL, a efecto de que el usuario no tenga que hacer largas filas para realizar alguna consulta.

11. Ubicación de una terminal de cómputo, que sea del acceso de todos los usuarios, a efecto de que realicen cualquier consulta de manera automatizada, referente a los diferentes servicios y trámites que deben realizar en el Departamento.

12. Renovación de la red de cómputo, dado que ya se encuentra absoleta, lo que hace muy lento el servicio que se brinda (matrícula, admisión, entre otros).

13. Adquisición de tecnología moderna para la dotación al estudiante del carne (chip inteligente). Se recomienda la búsqueda de fuentes de financiamiento externo (BCIE). Cabe señalar que este sistema la UCR ya lo tiene diseñado para implementarlo en 1999.

14. Ubicación de los servicios de cajas del Departamento de Gestión Presupuestario y Financiero, en el Depto de Registro, para cancelar todos los costos de los trámites que ahí se realizan.

15. Formular un programa de capacitación que contribuya al mejoramiento del servicio de atención que brinda el Departamento de Registro. Se sugiere que sea la Unidad de Capacitación y Desarrollo del Departamento de Recursos Humanos, quien desarrolle este programa en coordinación con las jefaturas de sección y la Dirección del De- partamento de Registro.

Se recomienda incluir temas como: El servicio al cliente (conceptos, calidad del servicio, satisfacción del cliente, la importancia de las actitudes positivas en el servicio al cliente, las necesidades y expectativas del cliente entre otros).

Relaciones Humanas

Motivación

Relaciones Públicas

\section{Propuesta de un programa de capacitación para los funcionarios del departamento de Registro de la Universidad Nacional}

\section{Presentación}

El Departamento de Registro ha realizado acciones tendientes al mejoramiento de la infraestructura y plataforma tecnológica, esto con la finalidad de fortalecer la calidad del servicio al usuario, sobre la base de una planificación estratégica.

Para esos efectos uno de los objetivos de mediano plazo es fortalecer la integración de los procesos de trabajo, bajo la visión de mejoramiento continuo hacia los usuarios. Por esa razón, es imprescindible que los funcionarios del Departamento de Registro conozcan todos los procesos, bajo una perspectiva sistémica que involucre principalmente la calidad del servicio.

Se sabe que esto es posible realizarlo con la exposición de los procesos y algunas charlas sobre servicio al cliente. Sin embargo, se quiere asegurar una respuesta a la integridad del proceso ya iniciado y asumido por el Departamento de Registro. Por eso, y en el sentido más estricto se plantea hoy la necesidad -con carácter urgente- de diseñar y programar, como iniciativa interna del mismo Departamento, actividades de capacitación orientadas a garantizar el mejoramiento continuo a los servicios que se ofrecen a los usuarios. 


\section{Justificación}

La capacitación es un programa más del subsistema de planificación administrativa, que a su vez forma parte del sistema de planificación universitaria. Así entonces, cualquier esfuerzo que se realice en el ámbito de la Universidad Nacional para comprender y mejorar los procesos administrativos, debe partir del conocimiento y la realidad de que la capacitación es el mejor medio para lograrlo.

En realidad, lleva a considerar una nueva forma de analizar y entender el proceso desde la óptica de sistematización y profesionalización, con el fin de lograr el cambio de -actitud- que se requiere en los funcionarios para el mejoramiento básico y productivo de los servicios que presta la institución, y por ende el Departamento de Registro.

\section{Formulación de política de mejoramiento y desarrollo del recurso humano}

Este Plan de Capacitación se fundamenta en las políticas institucionales que el Consejo Universitario estableció, según SCU 122998 del 20 de agosto de 1998, referente a los recursos humanos, que al respecto señala:

1. - Capacita en forma permanente sus autoridades de dirección administrativa y académico-administrativa, en aspectos de gestión y gerencia.

2. Garantiza de conformidad con los recursos institucionales condiciones laborales óptimas a sus funcionarios y de ascenso profesional y salarial sustentadas en la calidad, producción y dedicación sostenida.

3. Cuenta con un plan de inducción, actualización, capacitación, mejoramiento, formación y renovación de los recursos, congruente con la misión, los principios, los fines y las prioridades institucionales. La finalidad principal y los objetivos que persigue el programa deben ser:
Contribuir con el mandato institucional de promover el desarrollo y mejoramiento de los recursos humanos.

\section{Objetivos:}

1. Atender las necesidades profesionales de los funcionarios del Departamento de Registro, de acuerdo con las políticas institucionales.

2. Facilitar el desarrollo profesional de los funcionarios administrativos, de forma tal que mantenga su atención en las necesidades del usuario.

3. Lograr que el recurso humano que labora en la ventanilla del Depto. de Registro alcance motivación, que favorezca mejorar sus responsabilidades futuras, en función del servicio al usuario.

\section{Programa por módulos de capacitación}

Se entiende por módulos de capacitación el modelo integrado de temas formativos que buscan capacitar al recurso humano en aspectos como los conocimientos, habilidades y actitudes, con el objeto de mejorar su desempeño laboral.

El programa por módulos de capacitación es el conjunto de cursos que se impartirán a un grupo de personas, que tienen diferentes necesidades, ya sea para su actualización, desarrollo del puesto, desarrollo profesional y por los requerimientos del puesto,

De acuerdo con las características organizacionales que presenta el Departamento de Registro, estos programas se pueden plantear con la siguiente estructura:

\section{Módulo Disciplinario:}

Corresponde a una área específica de la administración, compuesta por una serie de cursos temáticos. Por ejemplo: El 
Área de Registro, podría integrar cursos sobre: Planificación estratégica, Servicio al Cliente, Relaciones Humanas, entre otros.

El propósito de este módulo es brindar una capacitación específica en una determinada área y se puede dirigir a todos los niveles.

\section{Diseño de Planes y Programas}

El Departamento de Registro diseñará los planes y programas, en coordinación con los jerarcas de la Unidad de Capacitación y Desarrollo y profesionales en el campo, además contará con la colaboración del instructor que asignará esta Unidad para ejecutar el determinado programa o curso.

Dicha coordinación, es para la planeación curricular de las actividades de capacitación y deben integrar aspectos tales como: los objetivos y contenido de los cursos, población meta (participantes), la disciplina a impartirse, el tiempo de duración, la prioridad, el material didáctico y de apoyo y la evaluación correspondiente.

Para los efectos de esta propuesta se ofrece como ejemplo la estructura por módulos disciplinarios.

\section{Módulo Disciplinario \\ Área de Registro}

\section{Módulo de Métodos y Procedimientos Administrativos}

Tiene como objetivo el ofrecer diferentes herramientas de orientación en las acciones y decisiones de los procesos administrativos. Contiene los siguientes temas:

Normativa General.

Liderazgo Participativo.

Procesos Internos.

Calidad Total.

Mecanismos de Coordinación.

Evaluación de Procesos.

Sistemas de Información.

\section{Módulo de Recursos Humanos}

Su propósito es desarrollar habilidades y destrezas en el accionar estratégico de la organización y la administración del recurso humano. El contenido del módulo es el siguiente:

\section{- Desarrollo Organizacional. \\ - Estrategias para la Superación Personal y Grupal. \\ - Comportamiento Organizacional. \\ - Convivencia Armoniosa en la Organi- zación. \\ - Motivación. \\ - Servicio al Cliente. \\ - El Cliente y su importancia. \\ - Reglas para brindar un óptimo Servicio. \\ - Imagen del Servicio que se desea Pro- yectar. \\ - Atención Personalizada.}

Los anteriores módulos tendrán como objeto el proporcionar al participante la oportunidad de consolidar los conocimientos, habilidades y actitudes fundamentales para mejorar su desempeño laboral.

\section{Organización y Desarrollo}

El objetivo de esta etapa es brindar una estructura general que le facilite a la Unidad de Capacitación y Desarrollo la ejecución de los planes y programas que se propongan. Por ejemplo se señala la siguiente variable, considerada como fundamental:

\section{Instructores}

La Universidad Nacional cuenta con recursos humanos administrativos y docentes capacitados y especializados en diversas disciplinas de los cuales se puede seleccionar y disponer como futuros oferentes instructores para coadyuvar al desarrollo de la propuesta.

En ese sentido, la Unidad de Capacitación y Desarrollo debe fomentar todos los 
mecanismos necesarios de coordinación con las autoridades universitarias respectivas para la preparación y actualización de los candidatos elegidos para tal efecto, entre los cuales pueden estar los jerarcas del Departamento de Registro, académicos de la Escuela de Secretariado Profesional, del Instituto de Estudios del Trabajo y de la Escuela de Planificación y Promoción Social.

Un equipo de instructores internos, ofrece a la Institución muchas ventajas y oportunidades, como: la reducción de costos en la contratación de instructores o empresas capacitadoras y la oportunidad de satisfacer oportunamente las necesidades específicas de capacitación. Asimismo, al contar con instructores internos experimentados laboralmente, el alcance del aprendizaje es más amplio y adaptable a las necesidades del recurso humano y los objetivos del Departamento de Registro.

\section{Ejecución}

El objetivo de esta etapa es llevar a la práctica los planes y programas de capacitación a través de los módulos establecidos de acuerdo a las acciones previstas. El éxito de esta etapa dependerá de la organización de las anteriores.

En esta línea la Unidad de Capacitación y Desarrollo mantendrá coordinación permanente con los instructores, con el fin de facilitar los medios necesarios para asegurar el logro de los objetivos propuestos.

Asimismo, crear los mecanismos de control necesarios para obtener información sobre los siguientes aspectos:

a) Controles de asistencia de los participantes e instructores, con el fin de que la Unidad de Capacitación y Desarrollo informe a la autoridad competente.

b) Sobre el desempeño y metodología de enseñanza de los instructores, esto con el propósito de corregir anomalías a tiempo que garanticen un mayor aprendizaje de los participantes. c) La evaluación del aprendizaje obtenido por el participante, esto con el fin de identificar el logro de eficiencia deseado o si es necesario complementar con actividades adicionales.

Finalmente, es importante recordar que el desarrollo de la presente propuesta debe llevarse a cabo en estrecha coordinación con las acciones de desarrollo institucional que realiza la Unidad de Capacitación y Desarrollo, esto permitirá aunar esfuerzos en procura de un mismo objetivo: retroalimentar las acciones de mejoramiento continuo que impulsa el Departamento de Registro.

\section{Referencias bibliográficas}

Berry Leonard L. A. Parasuraman. Marketing en las empresas de servicios. Compita mediante la calidad. Editorial Norma, México, 1995.

Gómez, Miguel. Elementos de estadística descriptiva. 2a. edición. Editorial EUNED. San José, Costa Rica, 1992.

Hamel, Gary Prahalad C.K. Compitiendo por el futuro. Estrategia crucial para crear los mercados del mañana. Editorial Ariel S.A. Barcelona España 1995.

INTECO. Gestión de la calidad y elementos de los sistemas de calidad. Lineamientos para los servicios. INTECO ISO 9004-2. Editorial Tecnología de Costa Rica. San José, Costa Rica.

Jurán J.M. Análisis y planeación de la calidad. 2a. Edición. Editorial MC Graw Hill. Madrid, España, 1994.

J.M. Jurán: Jurán y la Planificación de la Calidad. Ediciones Díaz de Santos S.A. Madrid, España, 1990. 
Koontz Harold. Weihrich Heinz Administración una perspectiva global. Novena Edición. Editorial McGraw Hill. México, 1993.

Sanabria Perera, Ronald. Calidad del servicio. Colegio de Médicos de Costa Rica, San José, Costa Rica, 1995.

Universidad Nacional. Plan de Trabajo, Departamento de Registro, 1999.

Estatuto Orgánico. Departamento de Publicaciones UNA, Heredia, Costa Rica, 1994.
Políticas Institucionales. Consejo Universitario. SCU-1229-98. Agosto 1998. Departamento de Publicaciones. UNA. 1998.

\section{Entrevistas}

Dr. Mainor Vargas. Vicerrector de DesarrolloUniversidad Nacional. julio-1998.

Lic. Blanca Amaya, Directora Departamento de Registro. Universidad Nacional. 